\title{
How do the small planetary satellites rotate?
}

\author{
Alexander V. Melnikov and Ivan I. Shevchenko \\ Pulkovo Observatory of the Russian Academy of Sciences, \\ Pulkovskoje ave. 65, St.Petersburg 196140, Russia \\ email: melnikov@gao.spb.ru, iis@gao.spb.ru
}

\begin{abstract}
We investigate the problem of the typical rotation states of the small planetary satellites from the viewpoint of the dynamical stability of their rotation. We show that the majority of the discovered satellites with unknown rotation periods cannot rotate synchronously, because no stable synchronous 1:1 spin-orbit state exists for them. They rotate either much faster than synchronously (those tidally unevolved) or, what is much less probable, chaotically (tidally evolved objects or captured slow rotators).
\end{abstract}

Keywords. Planets and satellites: general.

The majority of planetary satellites with known rotation states rotates synchronously (like the Moon, facing one side towards a planet), i.e., they move in synchronous spin-orbit resonance 1:1. The data of the NASA reference guide (NASA website data) combined with additional data (Maris et al. 2001; Maris, Carraro \& Parisi 2007; Grav, Holman \& Kavelaars 2003) implies that, of the 33 satellites with known rotation periods, 25 rotate synchronously.

For the tidally evolved satellites, this observational fact is theoretically expected. The planar rotation (i.e., the rotation with the spin axis orthogonal to the orbital plane) in synchronous 1:1 resonance with the orbital motion is the most likely final mode of the long-term tidal evolution of the rotational motion of planetary satellites (Goldreich \& Peale 1966; Peale 1977). In this final mode, the rotational axis of a satellite coincides with the axis of the maximum moment of inertia of the satellite and is orthogonal to the orbital plane.

Another qualitative kind of rotation known from observations is fast regular rotation. There are seven satellites that are known to rotate so (Maris et al. 2001; Maris, Carraro \& Parisi 2007; Grav, Holman \& Kavelaars 2003; Bauer et al. 2004; NASA website data): Himalia (J6), Elara (J7), Phoebe (S9), Caliban (U16), Sycorax (U17), Prospero (U18), and Nereid (N2); all of them are irregular satellites. These satellites, apparently, are tidally unevolved.

A third observationally discovered qualitative kind of rotation is chaotic tumbling. Wisdom, Peale \& Mignard (1984) and Wisdom (1987) demonstrated theoretically that a planetary satellite of irregular shape in an elliptic orbit could rotate in a chaotic, unpredictable way. They found that a unique (at that time) probable candidate for the chaotic rotation, due to a pronounced shape asymmetry and significant orbital eccentricity, was Hyperion (S7). Besides, it has a small enough theoretical timescale of tidal deceleration of rotation from a primordial rotation state. Later on, a direct modelling of its observed light curves (Klavetter 1989; Black, Nicholson \& Thomas 1995; Devyatkin et al. 2002) confirmed the chaotic character of Hyperion's rotation. Recent direct imaging from the CASSINI spacecraft supports these conclusions (Thomas et al. 2007).

It was found in a theoretical research (Kouprianov \& Shevchenko 2005) that two other Saturnian satellites, Prometheus (S16) and Pandora (S17), could also rotate chaotically (see also Melnikov \& Shevchenko 2008). Contrary to the case of Hyperion, possible chaos 
in rotation of these two satellites is due to fine-tuning of the dynamical and physical parameters rather than to a large extent of a chaotic zone in the rotational phase space.

We see that the satellites spinning fast or tumbling chaotically are a definite minority among the satellites with known rotation states. However, the observed dominance of synchronous behaviour might be a selection effect, exaggerating the abundance of the mode typical for big satellites. This is most probable. Peale (1977) showed on the basis of tidal despinning timescale arguments that the majority of the irregular satellites are expected to reside close to their initial (fast) rotation states.

A lot of new satellites has been discovered during last years. Now the total number of satellites exceeds 160 (see NASA website data). The rotation states for the majority of them are not known. In what follows, we investigate the problem of typical rotation states among all known satellites.

We consider the motion of a satellite with respect to its mass centre under the following assumptions. The satellite is a nonspherical rigid body moving in a fixed elliptic orbit about a planet. We consider the planet to be a fixed gravitating point. The shape of the satellite is described by a triaxial ellipsoid with the principal semiaxes $a>b>c$ and the corresponding principal central moments of inertia $A<B<C$. The dynamics of the relative motion in the planar problem (i.e., when the satellite rotates/librates in the orbital plane) are determined by the two parameters: $\omega_{0}=\sqrt{3(B-A) / C}$, characterizing the dynamical asymmetry of the satellite, and $e$, the eccentricity of its orbit. Under the given assumptions, the planar rotational/librational motion of a satellite in the gravitational field of the planet is described by the Beletsky equation (Beletsky 1965):

$$
(1+e \cos f) \frac{\mathrm{d}^{2} \theta}{\mathrm{d} f^{2}}-2 e \sin f \frac{\mathrm{d} \theta}{\mathrm{d} f}+\omega_{0}^{2} \sin \theta \cos \theta=2 e \sin f,
$$

where $f$ is the true anomaly, $\theta$ is the angle between the axis of the minimum principal central moment of inertia of the satellite and the "planet - satellite" radius vector.

As follows from an analysis of the Beletsky equation (see Melnikov \& Shevchenko (2000) and references therein), for a satellite in an eccentric orbit, at definite values of the inertial parameters, synchronous resonance can have two centres in spin-orbit phase space; in other words, two different synchronous resonances, stable in the planar rotation problem, can exist. Consider a section, defined at the orbit pericentre, of the spin-orbit phase space. At $\omega_{0}=0$, there exists a sole centre of synchronous resonance with coordinates $\theta=0 \bmod \pi, \mathrm{d} \theta / \mathrm{d} t=1$. If the eccentricity is non-zero, upon increasing the value of $\omega_{0}$, the resonance centre moves down the $\mathrm{d} \theta / \mathrm{d} t$ axis, and at a definite value of $\omega_{0}$ (e. g., for $e=0.1$ this value is $\simeq 1.26$ ) another synchronous resonance appears. Following Melnikov \& Shevchenko (2000), we call the former synchronous resonance (emerging at zero value of $\omega_{0}$ ) the alpha mode, and the latter one - the beta mode of synchronous resonance. Upon increasing the $\omega_{0}$ parameter, the alpha and beta modes coexist over some limited interval of $\omega_{0}$ (the extent of this interval depends on the orbital eccentricity), and in the section there are two distinct resonance centres situated at one and the same value of the satellite's orientation angle. Such a phenomenon takes place for Amalthea (J5) (Melnikov \& Shevchenko 1998; Melnikov \& Shevchenko 2000). On further increasing the $\omega_{0}$ parameter, at some value of $\omega_{0}$ the alpha resonance disappears, i. e., it becomes unstable in the planar problem, and only the beta resonance remains.

The " $\omega_{0}-e$ " stability diagram is presented in Fig. 1. Theoretical boundaries of the zones of existence (i.e., stability in the planar problem) of synchronous resonances are drawn in accordance with Melnikov (2001). Regions marked by "Ia" and "Ib" are the domains of sole existence of alpha resonance, "II" is the domain of sole existence of beta resonance, "III" is the domain of coexistence of alpha and beta resonances, "IV" is the domain of 
coexistence of alpha and period-doubling bifurcation modes of alpha resonance, "V" is the domain of non-existence of any 1:1 synchronous resonance, "VI" is the domain of sole existence of period-doubling bifurcation modes of alpha resonance.

The solid circles in Fig. 1 represent the satellites with known $\omega_{0}$. The open circles represent the satellites with the $\omega_{0}$ parameter determined by means of an approximation of the observed dependence of $\omega_{0}$ on the satellite size $r$, accomplished following an approach by Melnikov \& Shevchenko (2007). In total, the data on sizes and orbital eccentricities are available for 145 satellites (Karkoschka 2003; Sheppard \& Jewitt 2003; Sheppard, Jewitt \& Kleyna 2005; Sheppard, Jewitt \& Kleyna 2006; Porco et al. 2007; Thomas et al. 2007; JPL website data); so, there are 145 "observational points" in the stability diagram " $\omega_{0}-e "$ in Fig. 1. The horizontal bars indicate three-sigma errors in estimating $\omega_{0}$. They are all set to be equal to the limiting maximum value 0.21 .

From the constructed diagram we find that 73 objects are situated in domain $\mathrm{V}$, and in domain Ib 12 objects are situated higher than Hyperion (a sole solid circle in domain $\mathrm{Ib}$ ), while in domain Ib there are 15 objects in total. Synchronous state of rotation does not exist in domain V. For the majority of satellites in domain Ib (namely, for those that are situated higher than Hyperion) synchronous rotation is highly probable to be attitude unstable as in the case of Hyperion. So, 73 satellites in domain V and 12 satellites in domain Ib rotate either regularly and much faster than synchronously (those tidally unevolved) or chaotically (those tidally evolved). Summing up the objects, we see that a major part (at least 85 objects) of all satellites with unknown rotation states (132 objects), i.e., at least $64 \%$, cannot rotate synchronously.

In summary, though the majority of planetary satellites with known rotation states rotates synchronously (facing one side towards the planet, like the Moon), a significant part (at least $64 \%$ ) of all satellites with unknown rotation states cannot rotate synchronously. The reason is that no stable synchronous 1:1 spin-orbit state exists for these bodies, as our analysis of the satellites location on the " $\omega_{0}-e$ " stability diagram demonstrates. They

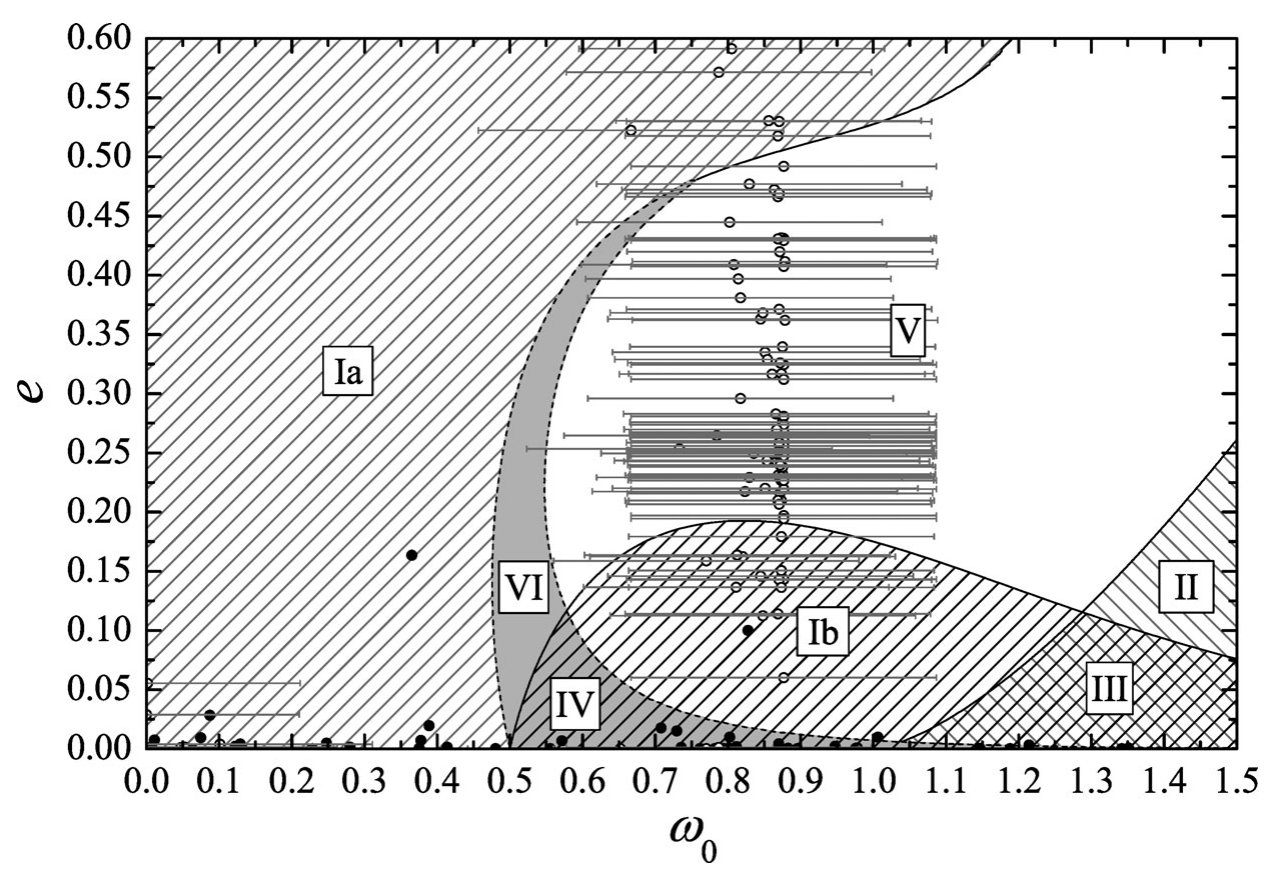

Figure 1. Location of the satellites with known radii in the " $\omega_{0}-e$ " diagram. 
rotate either regularly and much faster than synchronously (those tidally unevolved) or chaotically (tidally evolved objects or captured slow rotators).

With the advent of new observational tools, more and more satellites are being discovered. Since they are all small, they are all irregularly shaped (Kouprianov \& Shevchenko 2006). Besides, the newly discovered objects typically move in strongly eccentric orbits (see JPL website data; Sheppard \& Jewitt 2003). Therefore these new small satellites are expected to be located mostly in domain V of the " $\omega_{0}-e$ " stability diagram. Consequently, either fast regular rotation (most probable) or chaotic tumbling (much less probable), but not the ordinary synchronous 1:1 spin-orbit state, can be a typical rotation state for the newly discovered planetary satellites.

\section{References}

Bauer, J. M., Buratti, B. J., Simonelli, D. P., \& Owen, W. M. Jr. 2004, ApJ, 610, L57

Beletsky, V. V. 1965, The Motion of an Artificial Satellite about its Mass Center (Moscow: Nauka Publishers)

Black, G. J., Nicholson, P. D., \& Thomas, P. C. 1995, Icarus, 117, 149

Devyatkin, A. V., Gorshanov, D. L., Gritsuk, A. N., Melnikov, A. V., Sidorov, M. Yu., \& Shevchenko, I. I. 2002, Sol. Sys. Res., 36, 248

Goldreich, P. \& Peale, S. 1966, AJ, 71, 425

Grav, T., Holman, M. J., \& Kavelaars, J. J. 2003, ApJ, 591, L71

Karkoschka, E. 2003, Icarus, 162, 400

Klavetter, J. J. 1989, AJ, 98, 1855

Kouprianov, V. V. \& Shevchenko, I. I. 2005, Icarus, 176, 224

Kouprianov, V. V. \& Shevchenko, I. I. 2006, Sol. Sys.,Res. 40, 393

Maris, M., Carraro, G., Cremonese, G., \& Fulle, M. 2001, AJ, 121, 2800

Maris, M., Carraro, G., \& Parisi, M. G. 2007, A\& $A, 472,311$

Melnikov, A. V. 2001, Cosmic Res., 39, 68

Melnikov, A. V. \& Shevchenko, I. I. 1998, Sol. Sys. Res., 32, 480

Melnikov, A. V. \& Shevchenko, I. I. 2000, Sol. Sys. Res., 34, 434

Melnikov, A. V. \& Shevchenko, I. I. 2007, Sol. Sys. Res., 41, 483

Melnikov, A. V. \& Shevchenko, I. I. 2008, Celest. Mech. Dyn. Astron., 101, 31

Peale, S. J. 1977, in: J. A. Burns (ed.), Planetary Satellites, (Tucson: Univ. of Arizona Press), p. 87

Porco, C. C., Thomas, P. C., Weiss, J. W., \& Richardson, D. C. 2007, Science, 318, 1602

Sheppard, S. S. \& Jewitt, D. C. 2003, Nature, 423, 261

Sheppard, S. S., Jewitt, D., \& Kleyna, J. 2005, AJ, 129, 518

Sheppard, S. S., Jewitt, D., \& Kleyna, J. 2006, AJ, 132, 171

Thomas, P. C., Armstrong, J. W., Asmar, S. W., Burns, J. A., Denk, T., Giese, B., Helfenstein, P., \& Iess, L. 2007, Nature, 448, 50

Wisdom, J. 1987, AJ, 94, 1350

Wisdom, J., Peale, S. J., \& Mignard, F. 1984, Icarus, 58, 137

JPL website data, http://ssd.jpl.nasa.gov/ (Site Manager: D.K.Yeomans.)

NASA website data, http://solarsystem.nasa.gov/planets/ 